\title{
Multi-client Video Streaming over WirelessMAN-OFDMA
}

\author{
Vitor Bernardo* \\ Esa Piri ${ }^{\dagger}$ \\ * Laboratory of Communications and Telematics \\ Center for Informatics and Systems \\ University of Coimbra \\ Polo II, Pilhal de Marrocos, 3030-290 Coimbra, Portugal \\ \{vmbern, marilia\}@dei.uc.pt
}

Kostas Pentikousis ${ }^{\dagger}$

\author{
Marilia Curado* \\ ${ }^{\dagger}$ VTT Technical Research Centre of Finland \\ Kaitoväylä 1, FI-90571 \\ Oulu, Finland
}

Jarno Pinola ${ }^{\dagger}$

firstname.lastname@vtt.fi

\begin{abstract}
Wireless Interoperability for Microwave Access (WiMAX) is a prime contender in the Broadband Wireless Access (BWA) market due to its next generation network architecture, low patent portfolio load considerations, support for Quality of Service (QoS), and last but not least, high capacities. Although until recently there has been a lot of interest in the mobility support of the technology, with the recent push for the Third Generation Partnership Project's (3GPP) Long Term Evolution (LTE), vendors are advancing WiMAX (in particular with the Wireless Metropolitan Area Network - Orthogonal Frequency Division Multiple Access (WirelessMAN-OFDMA) air interface specified by the IEEE 802.16 working group) as a fixed broadband replacement. For this market segment, Audio/Video (A/V) delivery is expected to be a key service offering. In this paper, we perform a detailed empirical evaluation using a state-of-the-art WiMAX testbed with multiple video flows directed at several Customer Premises Equipment (CPE) devices simultaneously. We employ the Evalvid framework and develop a methodology for its use in multi-client evaluations. We report our results for Variable Bit Rate (VBR) and Constant Bit Rate (CBR) video streams and draw conclusions.
\end{abstract}

\section{Categories and Subject Descriptors}

C.2.5 [Computer-Communication Networks]: Local and WideArea Networks; C.4 [Performance of Systems]: Measurement techniques

\section{General Terms}

Measurement, Performance, Experimentation.

\section{Keywords}

Network Measurements, WiMAX, IEEE 802.16, WiMAX testbeds, video streaming, video quality assessment.

Permission to make digital or hard copies of all or part of this work for personal or classroom use is granted without fee provided that copies are not made or distributed for profit or commercial advantage and that copies bear this notice and the full citation on the first page. To copy otherwise, or republish, to post on servers or to redistribute to lists, requires prior specific permission and/or a fee.

PM2HW2N'09, October 26, 2009, Tenerife, Canary Islands, Spain.

Copyright 2009 ACM 978-1-60558-621-2/09/10...\$10.00.

\section{INTRODUCTION}

Broadband communications are being deployed on a multiplicity of locations, from large and dense cities to remote and sparse rural areas. More recently, Broadband Wireless Access (BWA) has extended the fixed infrastructure, aiming at a state where users will be connected anytime, anywhere, while reducing the installation costs inherent to fixed networks. Wireless Interoperability for Microwave Access (WiMAX) is a key technology for such scenario, due to its large range and high data rates capabilities, as well as for the native support of mobility and Quality of Service (QoS). There are two standards supporting WiMAX, namely, the IEEE 802.16d-2004 [1], for fixed wireless communications and the IEEE 802.16e-2005 [2], which supports mobile wireless communications.

The native characteristics of WiMAX, both in the fixed and mobile versions, allow the support of a large number of users, which, as it is becoming the trend, will extensively use multimedia applications, such as Voice over Internet Protocol (VoIP), Video on Demand (VoD) and IP Television (IPTV). This type of utilization poses important challenges, both in terms of the quality of transmission of the traffic generated by such applications and on the capability of the WiMAX link to support multiple users, which are using a variety of applications.

In recent years, researchers have addressed the capabilities of WiMAX to support multimedia applications resorting mainly to simulation studies, in particular with the mobile WiMAX evaluations. Although these efforts have allowed the characterization of WiMAX based on both IEEE 802.16d and IEEE 802.16e, and have also shown how multimedia applications behave when traversing WiMAX links, there is still a lack of practical evaluation. This gap is particularly stringent when it comes to the evaluation of this BWA technology to deliver multiple client - multiple flow video streaming traffic.

This paper uses a state-of-the-art WiMAX system to investigate the performance of the Wireless Metropolitan Area Network Orthogonal Frequency Division Multiple Access (WirelessMANOFDMA) air interface when delivering video streaming traffic. The contributions described are threefold. First, multiple Customer Premises Equipment (CPE) devices are used in a real WiMAX testbed suited for fixed and mobile deployments. Second, the capability of the WiMAX link to support multiple video streams from multiple clients is evaluated when the videos are compressed in three different ways, namely, using Constant Bit Rate (CBR) with 256kbps and 512kbps, and Variable Bit Rate 
(VBR) with $540 \mathrm{kbps}$ peak rate. By creating scenarios where the amount of active videos is increased, it is possible to assess the scalability capability of the WirelessMAN-OFDMA air interface related to the different compression levels used. Third, a novel evaluation methodology that accurately measures the network and user level performance of multiple video streams from multiple clients is proposed and applied to the practical study carried out. The proposed methodology consists in the definition of a staggered starting time for the video streams, in order to avoid client buffer overflow, and in the selection of the samples from each user to consider when to gather performance statistics, in order to guarantee that performance is evaluated when all the clients have active video streams.

The remaining sections of this paper are organized as follows. Section 2 describes related work on the evaluation of multimedia applications over WiMAX. The methodology used for the experimental evaluation is presented in Section 3, followed by the analysis of the results obtained in Section 4. Finally, Section 5 presents the main conclusions drawn from the extensive analysis and experimentation performed.

\section{RELATED WORK}

As WiMAX is foreseen to compete in the mobile BWA markets capitalizing on its salient features such as support for high peak data rates and built-in QoS support, new service models focusing on the delivery of Audio/Video (A/V) content are among the most interesting and promising usage scenarios for the technology. Hence, different multimedia applications such as VoIP, VoD and IPTV have been a central topic of investigation in both simulation and empirical WiMAX studies in recent years. WiMAX is often cited as the main alternative for fixed broadband replacement. Our interest is to investigate the performance of the WirelessMAN-OFDMA air interface in practice and measure its performance at capacity traffic levels with several simultaneous clients accessing the wireless network. We used a state-of-the-art WiMAX system suitable for both fixed and mobile deployments and explored its capability to deliver video streaming traffic. Consequently, in this section, we review previous studies focusing on VoD and video streaming over WiMAX. We stress, however, that our study employs multiple video streams over several CPE units inside the same mobile WiMAX cell.

Hillestad et al. [3] study the delivery of VoD content over fixed WiMAX links in rural areas where last mile deployments are currently the dominating method to use WiMAX technology. They perform NCTUns [4] simulations with different PHY layer modulation and coding schemes, ranging from BPSK $1 / 2$ to 16QAM 3/4, in order to determine the performance of the wireless link at different distances from the Base Station (BS) which is operating in the $3.5 \mathrm{GHz}$ frequency band in a Point to Multipoint (PMP) topology. The simulated air interface is using an Orthogonal Frequency Division Multiplexing (OFDM) with 20 $\mathrm{MHz}$ bandwidth and has 200 of its 256 subcarriers dedicated to user data transmission using 4:1 downlink/uplink ratio. The authors chose this ratio as a suitable configuration for a BS that is delivering (mainly) VoD services. Note, however, that in other studies, as well as in many commercial deployments, the typical downlink/uplink ratio is $2: 1$.

By observing the state of the video playout buffers at the receiving clients over a lossless and otherwise empty link with stable delays, the simulations in [3] aim to validate the maximum number of clients the WiMAX link can support in ideal conditions such that there are no quality impairments in the video playback. With this setup, Hillestad et al. come to the conclusion that 10 uniformly distributed clients can receive the VBR video stream with an average target rate of $750 \mathrm{~kb} / \mathrm{s}$ without difficulties while maintaining a very good Peak Signal to Noise Ratio (PSNR) level between the transmitted and received video signals. The authors also present a feedback-based adaptive video transmission scheme, which adjusts the transmitted video quality at the video server according to the playout buffer state at the receiving clients. The evaluation results obtained by using this method show gains in terms of decreased quality fluctuations of the received video.

Sousa et al. [5] use ns-2 [6] simulations in order to observe the performance of a mobile WiMAX link at vehicular speeds with real time multimedia content delivery scenarios. Their aim is to verify the negative effects of unprepared hard handovers that cause problems for real time traffic in mobility, as well as to validate the gains achievable with predictive handover preparation through the IEEE 802.21 Media Independent Handover (MIH) [7] framework. The simulation results show the clear gain of using cross-layer information in the handover preparation phase which due to the shorter disconnection times during the handover execution considerably increases the overall perceived quality of the received video content and, thus, the Quality of Experience (QoE) of the end user in mobile usage scenarios.

With respect to testbed measurement studies, Durantini et al. [8] use fixed WiMAX equipment operating in the $2.5 \mathrm{GHz}$ frequency band and perform a series of empirical measurements over their testbed in Rome, Italy, including $\mathrm{VoD}$ and video streaming services. The testbed equipment deploys OFDM PHY layer in the downlink with a $5 \mathrm{MHz}$ bandwidth and uses Time Division Duplexing (TDD) with 2:1 downlink/uplink ratio. The link span in the video delivery evaluations is 640 meters and it is located in an urban area. With these settings, the measured maximum throughput of the WiMAX link is approximately $8.1 \mathrm{Mb} / \mathrm{s}$ and 3.6 $\mathrm{Mb} / \mathrm{s}$ in downlink and uplink, respectively, when Ethernet Maximum Transmission Unit (MTU) sized User Datagram Protocol (UDP) packets are used with 64QAM 3/4 modulation. Based on this measured capacity, the authors set the number of video flows in the test so that both the downlink and uplink are operating at full capacity when two separate CPEs are receiving the video content.

By studying the impact of different QoS classes and service differentiation on the VBR video streaming performance of the testbed, Durantini et al. validate the role of WiMAX QoS classes in the stability of the provided video delivery service. The main comparison is done between the Real-Time Polling Service (rtPS) scheduling for video streaming and Non-Real-Time Polling Service (nrtPS) scheduling for VoD. The measurements clearly show that rtPS should be used for real time video delivery in commercial deployments if preplay buffering is not used at the receiving client.

In previous studies at the VTT Converging Networks Laboratory, we have empirically evaluated video delivery using synthetic traffic generation in a fixed WiMAX testbed deploying a WirelessMAN-OFDM air interface with a maximum goodput of $9.4 \mathrm{Mb} / \mathrm{s}$ and $5.5 \mathrm{Mb} / \mathrm{s}$ in the downlink and uplink, respectively 
[9][10]. In particular, the aim has been to investigate the behavior of the WiMAX link in saturation when mixed multimedia traffic consisting of VoIP and video streams is transmitted over the air interface from the BS to two separate receiving CPEs. The used testbed equipment is using Frequency Division Duplexing (FDD) with a $3.5 \mathrm{MHz}$ channel bandwidth and 2:1 downlink/uplink ratio at the $3.5 \mathrm{GHz}$ frequency band. By using the 64QAM 3/4 modulation in Line of Sight (LOS) conditions, the downlink can handle $5-8$ VBR video streams encoded with the H.264/AVC [11] codec, also known as MPEG-4 Part 10, and transmitted at a data rate of approximately $512 \mathrm{~kb} / \mathrm{s}$, even when the link is already delivering VoIP streams with its full capacity. The additional bandwidth for video streaming is available due to the heavily suboptimal capabilities of the used WiMAX equipment to handle small VoIP packets. In Non-Line of Sight (NLOS) conditions, the number of simultaneous good quality video streams decreases to 1 -4 when a similar scheme is used. The modulation used in NLOS measurements is 16QAM 3/4. Video quality is evaluated by using theoretical threshold values for both packet loss and one-way delay. In both LOS and NLOS cases, an additional CBR audio stream accompanying each of the video steams is transmitted separately at $192 \mathrm{~kb} / \mathrm{s}$.

Recognizing a gap in the previously published results on video delivery over WiMAX, this paper aims to provide new knowledge on the behavior of state-of-the-art WiMAX equipment in usage scenarios, which include congested links with multiple users and no QoS differentiation of any kind as a countermeasure for the decreased capacity available for individual subscribers. By using a testbed setup and empirical evaluations instead of simulations, no assumptions on the air interface behavior and parameters have to be made. In addition, the testbed deployment comprising a BS and four separate CPEs with varying capabilities, strives to emulate a real life usage scenario as accurately as possible in our testbed set-up. Accordingly, our empirical results correspond directly to what one would expect to measure in a deployed commercial WiMAX network. By introducing a video quality assessment tool into the testbed, the actual application layer effects caused by the air interface can also be observed in detail.

\section{METHODOLOGY}

This section presents the overall scenario used for the evaluation of video streaming over mobile WiMAX with multiple CPEs. First, the WiMAX testbed configuration is described, including the characteristics of the used equipment and the testbed topology. Second, the traffic generation tool and its integration within the WiMAX testbed are specified. Finally, a novel and accurate evaluation methodology for the correct evaluation of multi-CPE / multi-video sessions is proposed.

In our measurements, four CPEs are connected to one BS using 5 $\mathrm{MHz}$ channel bandwidth and the best effort QoS scheme. Two of the CPEs are Zyxel MAX-210M1 supporting modulation scheme of 64QAM 1/2 in the downlink. Another two are Accton RG230 CPEs employing 64QAM 5/6 in the downlink. Uplink modulation employed by all CPEs is 16QAM 3/4. All the end-hosts and the video servers run on GNU/Linux operating systems (Ubuntu 8.04.2, kernel 2.6.24-24).

\subsection{Testbed}

The schematic of the mobile WiMAX tested employed in our experimental evaluation is illustrated in Figure 1. The fully configurable testbed is located at the VTT Converging Networks Laboratory ${ }^{1}$ comprising two Alcatel-Lucent $9710 \mathrm{C}$-WBS base stations BSs operating on the $3.5 \mathrm{GHz}$ frequency band.

Before proceeding with the evaluation we measured the maximum throughput that can be attained with our testbed facilities. The maximum attained downlink throughput over the testbed was $9.3 \mathrm{Mb} / \mathrm{s}$, using the 64QAM $5 / 6$ modulation scheme, $5 \mathrm{MHz}$ channel bandwidth, and MTU of 1400 bytes.

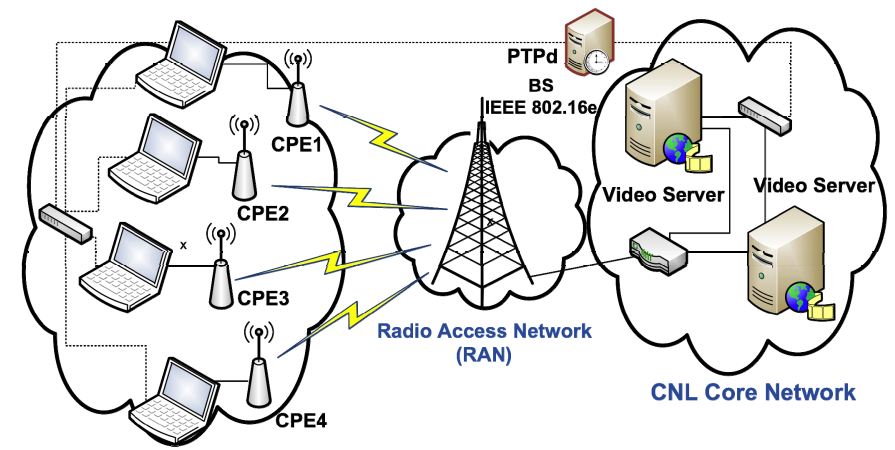

Figure 1: Mobile WiMAX testbed architecture

In order to measure one-way end-to-end delay, system clocks of the end-hosts need to be accurately synchronized. To attain synchronization with an adequate level of precision we opted to use PTPd [12], an open-source implementation of the IEEE 1588 Precision Time Protocol (PTP) [13]. PTPd provides synchronization accuracy in the magnitude of sub-milliseconds, with coordination in the order of $10 \mu \mathrm{s}$ [14]. All end-hosts use a dedicated network card for the exchange of PTP messages, which assures that the synchronization traffic does not introduce any overhead on the WiMAX link.

\subsection{Traffic Generation}

The Evalvid framework was chosen to perform the traffic generation as well as the offline video evaluation [15]. One of the main motivations to use the Evalvid framework over other video quality measurement tools is its ability to reconstruct the received video, even without some missing frames (lost in the network) and to measure its quality by performing a comparison with the original video.

The Evalvid framework is able to report different useful parameters in both network and video quality analysis perspectives. On one hand, it is possible to obtain, for instance, the packet loss and the one-way delay and jitter metrics. On the other hand, metrics based on the end-user perceived quality, also known as QoE, such as Mean Opinion Score (MOS) [16] are also available.

Figure 2 presents the Evalvid framework. The Video Server entity is responsible for the startup of the video streaming to the enduser through the network. For a detailed description of the Evalvid framework please see [15]. In brief, the Evalvid framework works as follows. In the first step, the video preparation is performed; a raw lossless video is compressed in order to be sent to the receiving end-user. Then, the sender is able to start the streaming, and simultaneously, it will begin to capture useful information of the transmitted video. Details such as frame

\footnotetext{
${ }^{1}$ http://www.cnl.fi
} 
types and sending times are collected with the Evalvid sending tool, and the well-know tcpdump tool collects the network information about the transmitted packets. The analogue network capture is repeated in the receiver side until the video transmission ends, however, the information about the video itself is not captured.

Then, when the information has been completely collected in both the sender and the receiver, the framework is able to reconstruct the video at the receiver by using the collected information and the original transmitted video file.

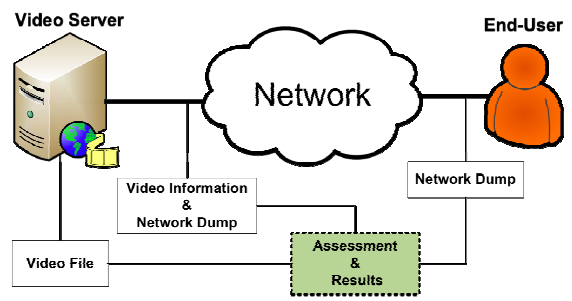

Figure 2: Simplified diagram of the Evalvid framework

In the next step, the reconstructed coded video is transformed back into raw format, in order to perform the video quality comparison with the original lossless raw video. The comparison is performed through the calculation of the PSNR between the reconstructed and the original lossless raw video. As a result of this assessment, the framework will output the requested metrics. The MOS metric is calculated by having the PSNR as reference and using a well-know relationship between the two metrics, as shown in Table 1.

Table 1: PSNR to MOS conversion

\begin{tabular}{|c|c|l|l|}
\hline PSNR & MOS & Perceved Quality & \multicolumn{1}{|c|}{ Impairment } \\
\hline$<20$ & 1 & Bad & Very annoying \\
\hline $20-23$ & 2 & Poor & Poor annoying \\
\hline $25-31$ & 3 & Fair & Slightly annoying \\
\hline $31-37$ & 4 & Good & Perceptible, but no annoying \\
\hline$>37$ & 5 & Excellent & Imperceptible \\
\hline
\end{tabular}

\subsection{Tests Description}

The tests were performed using the well-known Akiyo video, which is a lossless raw movie with 300 frames. Figure 3 presents the first frame of this video.

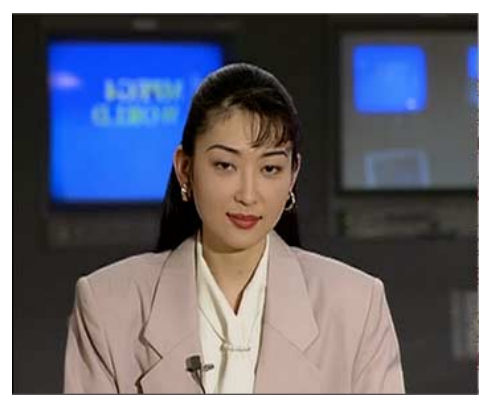

Figure 3: Sample frame from the Akiyo movie

The video used in this evaluation was compressed with the MPEG-4 [17] codec. This state-of-the-art codec uses three different types of frames, namely, I-frames, P-frames and Bframes. The only independent frames are I-frames. P-frames are predicted frames and depend on the previous I-frame. B-frames are bidirectional predicted frames and depend on both I-frames and P-frames. The only optional frames are the B-frames. Moreover, the MPEG-4 organizes all frames in a Group of Pictures (GOP), which consists of a single I-frame, various Pframes and, optionally, the corresponding B-frames.

The Akiyo video used to perform the evaluation was compressed in three distinct ways: CBR of both $256 \mathrm{kbps}$ and $512 \mathrm{kbps}$ rates and a VBR, which has a peak rate of 540kbps. These videos are, respectively, referred during the next sections as CBR-256kbps, CBR-512kbps and VBR. Moreover, all movies were compressed using a GOP of 30 frames with 25 Frames Per Second (FPS).

All the presented values in the following sections are based on multiple runs, in order to obtain more accurate results.

\subsection{Evaluation Methodology}

The evaluation described in this work uses multiple CPEs simultaneously and each CPE supports several concurrent video streaming flows. Unfortunately, the Evalvid framework is developed and also mainly used for single-client/single-flow evaluation scenarios. In order to overcome this shortcoming, we developed a new methodology to take advantage of the rich evaluation toolbox that Evalvid provides.

The easiest solution would be to start all the flows simultaneously. In other words, we could inject the traffic corresponding to multiple users using Evalvid all at once, sending several movies to the network exactly at the same time. However, with this approach, buffer overflows would occur at the network interfaces of the senders, causing immediate packets losses which are unrelated to the WiMAX link capability.

To overcome this limitation, an evaluation methodology was developed that is able to deal with the buffer overflow problem by adopting a staggered starting time schedule, which roughly corresponds to service flow activations in a real deployment. With our methodology, it is assured that all analyzed samples from each user are taken at playout time when all the users are effectively playing a video. Thus, the video quality perceived by the end-users as well as the conventional network parameters can be accurately evaluated. Figure 4 depicts the model used in the methodology.

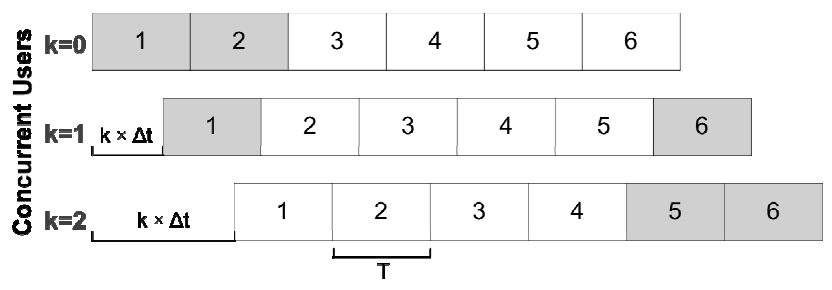

Figure 4: Multiple client evaluation methodology schema

Each block represents a video with a certain playtime $(\mathrm{T})$. At a first stage, the initial bottleneck identified above is avoided by spacing the instant when the clients start playing a video, using a time interval $(\Delta \mathrm{t})$ between each client. Then, at a second stage, there is the need to select the videos of each client $(\mathrm{k})$, which will 
be evaluated. These videos must all be running videos when all the clients are active.

In Figure 4, the white blocks represent the videos that can be used in the evaluation process and the grey blocks those to be discarded. Equation 1 is defined to calculate the sequence number of the first useful video of each client $\mathrm{k}$.

$$
M(k)=\left\lceil\frac{(N-k) \times \Delta t}{T}\right\rceil+1
$$

where:

$$
\begin{aligned}
& N=\text { Total number of videos } \\
& k=\text { Current client } \\
& \Delta t=\text { Start delay between each client } \\
& T=\text { Single video time }
\end{aligned}
$$

Moreover, the Equation 1 was extended to enable the calculation of the minimal number of video repetitions required to grant a certain desired number of useful samples in all clients. This extension is shown in Equation 2.

$$
R(c)=M(0)+c
$$

where:

$$
c=\text { Desired number of samples }
$$

All the results presented in the following sections will use this methodology.

\section{EXPERIMENTAL EVALUATION}

This section presents the main goals of the performed experimental testbed evaluation and discusses the obtained results.

\subsection{Objectives}

The main objective of this evaluation is to thoroughly quantify empirically the capacity of WirelessMAN-OFDMA links to support multiple simultaneous and independent video flows to several subscribers. The assessment of these capabilities will take into account both conventional network parameters, such as oneway delay and frame loss, as well as QoE metrics such as MOS.

The followed approach was the "stress test", which starts with a low number of concurrent users, $N$, and then gradually increases it according to the evaluation methodology presented in the previous section.

\subsection{Results}

This section presents the results of the performed tests.

\subsubsection{One-way Delay}

Figures 5-7 illustrate the end-to-end one-way delays (OWD) for the CBR-256kbps, CBR-512kbps and the VBR movies, respectively. The $\mathrm{x}$-axis represents the number of concurrent video streams in the network and the y-axis the end-to-end $O W D$ in milliseconds. In these box plots, the red line inside the box is the mean $O W D$ and the red crosses represent the outliers, i.e, the values that are not between the first and the third quartiles.

As shown in Figure 5, the mean $O W D$ when the CBR-256kbps video is playing stays below $25 \mathrm{~ms}$ up to $N=30$ simultaneous concurrent video streams. From that value onwards, the $O W D$ begins to increase until it reaches $50 \mathrm{~ms}$ for $N \leq 44$. Moreover, with this video data rate, for all values of $N$, it is possible to guarantee that $75 \%$ of all concurrent clients will have an $O W D \leq$ $100 \mathrm{~ms}$, as shown by the third quartile values plotted in the figure (i.e. the upper limits of the blue boxes).

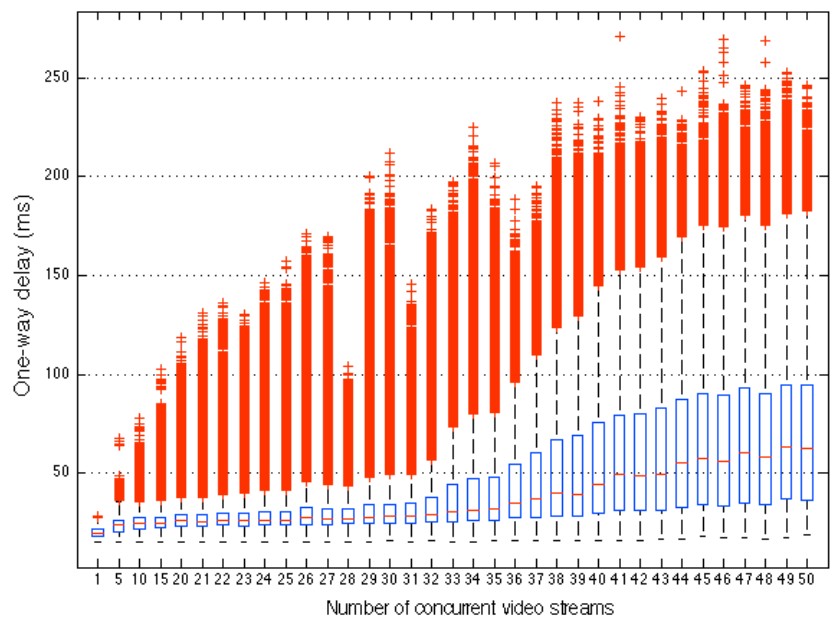

Figure 5: CBR-256kbps video one-way end-to-end delay

Figure 6 shows that when the CBR-512kbps video is employed, the $O W D$ results are not as good as above. In this case, to keep the $O W D$ below $25 \mathrm{~ms}$, the number of simultaneous video streams must be below 13 . However it is only possible to ensure an $O W D$ $\leq 100 \mathrm{~ms}$ for $75 \%$ of all $N$ clients when $N<25$. This is one half of the $N$ simultaneous users supported with the CBR-256kbps video.

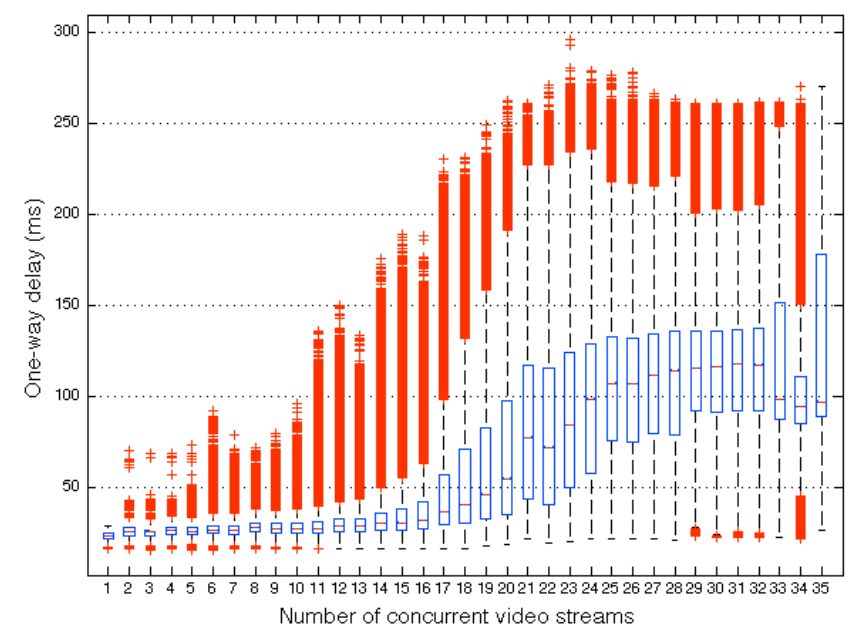

Figure 6: CBR-512kbps video one-way end-to-end delay

The behavior of the $O W D$ when using the VBR video, as shown in Figure 7, is comparable to the previously presented CBR$512 \mathrm{kbps}$ video. Nonetheless, in the CBR-512kbps case, it is possible to ensure an $O W D \leq 25 \mathrm{~ms}$ for $N<19$ and, with the VBR video this value is already reached for $N<17$. However, the VBR video has a better performance than CBR-512kbps for higher values of $N$, such as $N>30$. In these cases, the usage of a VBR video guarantees a better $O W D$ in almost all the cases, due to the bit rate variation. 


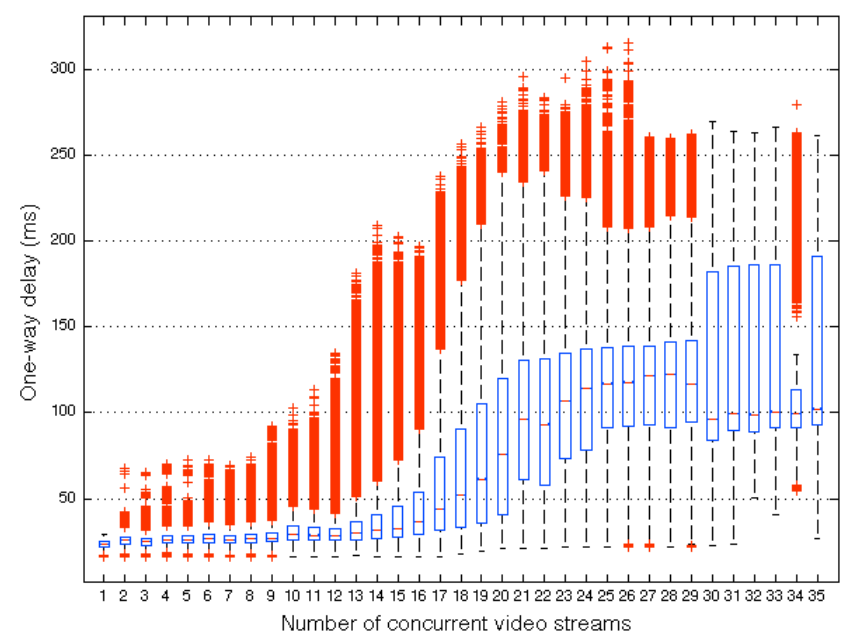

Figure 7: VBR video one-way end-to-end delay

As expected, the maximum $O W D$ values, as well as the majority of the high outlier values, is worst in the cases where the network has a higher load, such as $N>45$. In these scenarios, the maximum $O W D$ can reach $250 \mathrm{~ms}$. However, even in these situations almost all the clients will have an $O W D<100 \mathrm{~ms}$.

Concisely, these results show that WiMAX possesses the required capabilities to ensure an acceptable $O W D$ for multiple simultaneous video streams. It is possible to encompass an $O W D$ $<50 \mathrm{~ms}$ for $N<17$ for both CBR-512kbps and VBR videos. With a CBR-256kbps video similar $O W D$ values are achieved when $N$ $<43$. These results clearly show that the WiMAX link can support twice as many clients without increasing the $O W D$ in the same proportion.

\subsubsection{Frame Loss}

Figure 8 shows the complete frame loss rate for each simultaneous stream in the all tested videos. The y-axis shows the complete frame loss percentage and the $\mathrm{x}$-axis is the number of concurrent video streams currently traversing the network.

The complete frame loss percentage is calculated based on the packet loss information collected from the network. Then, the Evalvid framework will match the packets and the correspondent frames with the help of the frame information file created in the beginning of the experimentation. This data is then used to discard the frames which have some packets missing.

The complete frame loss rate provides a better measure of the quality perceived by the end-user when compared with the packet loss, because it will take into account the codec characteristics. As stated before, the MPEG-4 codec is composed by three different types of frames, which are related and dependent of each other. So, for instance, if a I-frame is lost, it is possible that the entire GOP may be compromised as well, since the lost I-frame will make all dependent frames useless.

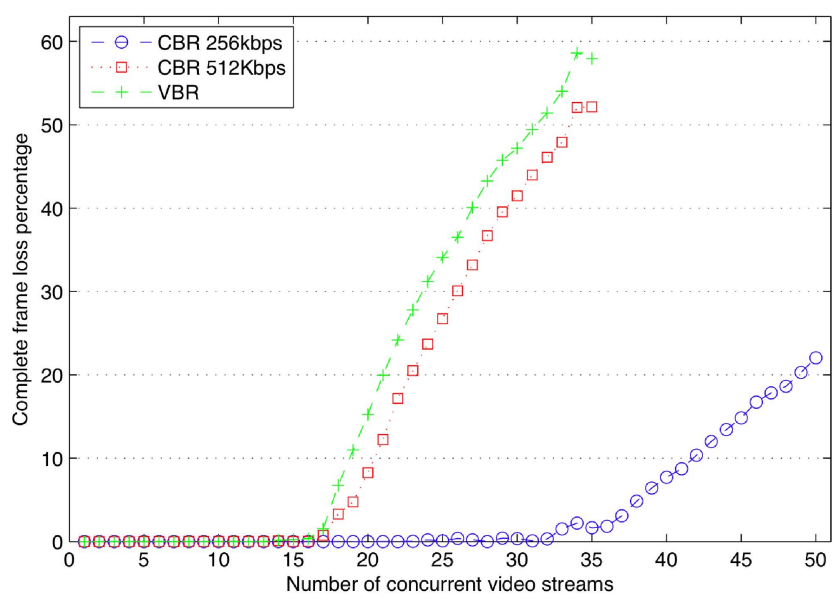

Figure 8: Complete frame loss rate

By using either CBR-512kbps or VBR compression, the frame loss percentage is always below $1 \%$ for $N \leq 16$ clients. The same behavior can be observed for CBR-256kbps, up to $N \leq 32$ clients. After this threshold, the frame loss rate slowly increases, as the WiMAX BS is not using any retransmission recovery protocol, such as Automatic Repeat-reQuest (ARQ).

The packet loss percentage for each video is depicted in Figure 9. As expected, the packet loss percentage behavior follows a similar trend to the complete frame loss percentage, because they are correlated, i.e, the complete frame loss rate is calculated through the packet loss information.

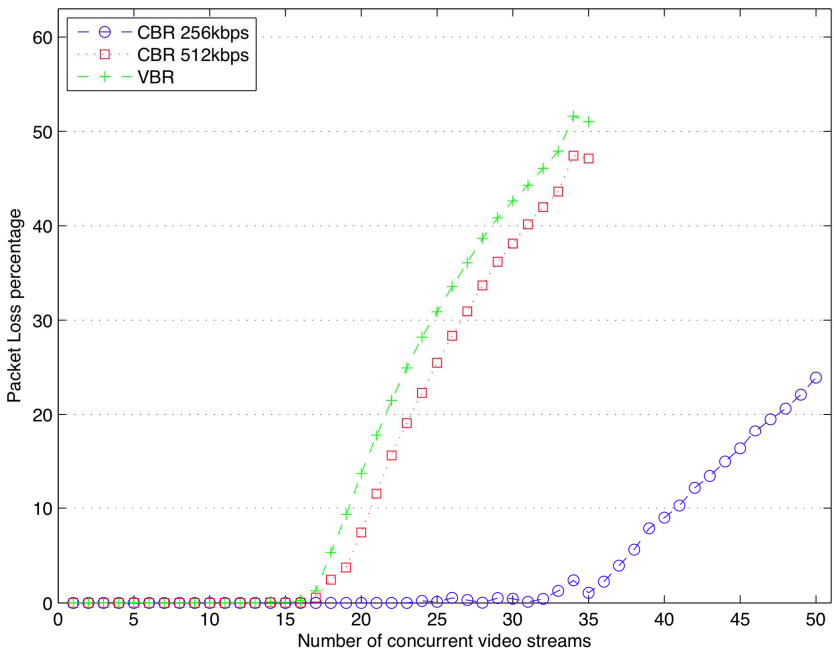

Figure 9: Packet loss rate

For the VBR video with $N=34$, the difference between the complete frame loss rate and the packet loss rate is almost $10 \%$. This means that some frames are almost completely transmitted, but since there are some packets missing, they will be marked as lost frames. A similar behavior is observed in the CBR-512kbps video. However, with this compression rate, the gap between the packet loss rate and frame loss rate is lower $(5 \%)$.

When the CBR-256kbps video is playing there is no significant discrepancy between the packet loss and the frame loss. This means that in this case there are not too many frames marked as lost due to only few missing packets. 


\subsubsection{Mean Opinion Score}

Figure 10 depicts the relationship between the number of simultaneous clients and the MOS. The line corresponding to the MOS value of 3 represents a video that the end-user considers as having a fair quality. The higher MOS values correspond to the best quality, where a MOS of 5 is excellent, and lower MOS values indicate poor to bad quality $(\mathrm{MOS}=1)$.

The Evalvid framework does the MOS assessment through the PSNR information with the mapping previously presented in Table 1

The employed videos have a distinct initial MOS due to the different compression rates. The CBR-256kbps has an initial MOS of 4.8 and both CBR-512kbps and VBR versions have maximum MOS, i.e, 5 .

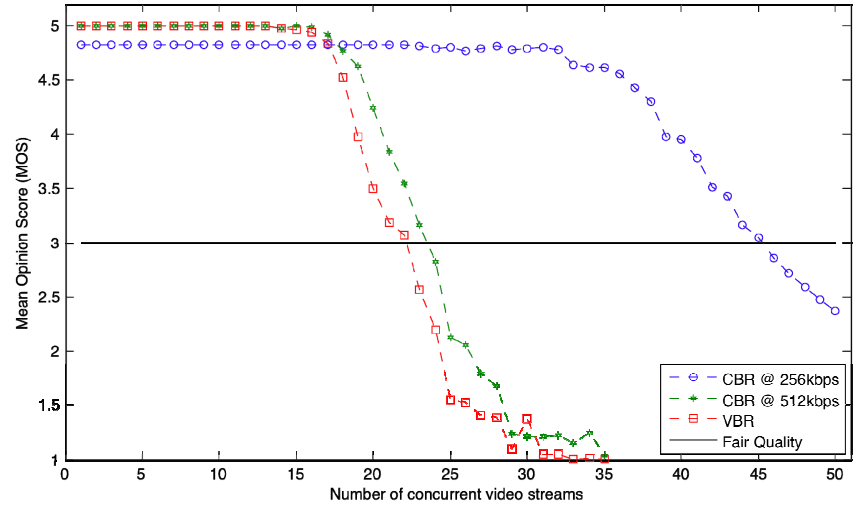

Figure 10: Mean Opinion Score (MOS)

All videos have a very good perceived quality up to $N=17$ clients whilst from that value onwards, the end-users estimated opinion about the video quality goes slightly down. Nevertheless, it is possible to ensure a reasonable quality up to $N=22$ concurrent streams. The quality of all the videos is similar when the network load is low. However the VBR behavior is the worst when the WiMAX link becomes more congested. This is clearly perceived by the end-users, especially, when the quality is near the fair borderline.

The CBR-256kbps video is not capable of providing a MOS value of 5 (maximum), due to the initial quality restrictions of the video compression rate. However, while using this version of the video, the WiMAX segment can support $N \leq 36$ clients with a good quality, as illustrated by the MOS greater than 4 . Moreover, for $N$ $\leq 45$ clients it is still possible to ensure a pleasant experience to all the clients, with the MOS always above the fair quality.

The number of well-supported users using the CBR-256kbps is roughly twice the value of users sustained with both CBR$512 \mathrm{kbps}$ and VBR videos. Observing these values we can conclude that in our testbed, with four different CPEs and one WiMAX BS, it is possible to support more clients if the initial video bit rate is lower, such as the CBR-256kbps video.

The measured maximum downlink bandwidth of the testbed BS with a 64QAM 5/6 modulation is $9.3 \mathrm{Mb} / \mathrm{s}$. On the other hand, the minimal bandwidth required to transport a CBR-256kbps video for $N=45$ in perfect conditions is approximately $11.52 \mathrm{Mb} / \mathrm{s}$. This is more than $2 \mathrm{Mb} / \mathrm{s}$ higher than the maximum attained downlink capacity. Hence, it is possible to conclude that the WiMAX segment is able to provide an acceptable quality for end-users, even when the maximum empirical throughput is exceeded.

In brief, the MOS reflects the behavior of the end-to-end $O W D$ and the complete frame loss, as expected. Figure 10 shows that by using the CBR-256kbps video it is possible to guarantee a good quality for twice as many concurrent streams than with both the CBR-512kbps and VBR versions. Moreover, these results highlight the capability of WiMAX using the WirelessMANOFDMA air interface to support multi-user video streaming in limit conditions, such as when the bandwidth requirements from the clients exceed the empirically measured maximum capacity of the BS.

\section{CONCLUSION}

The successful deployment of WiMAX as a BWA technology will be strongly dependent on how it will support multiple users and how it will be able to distribute multimedia applications such as VoIP, VoD and IPTV. In particular, the performance of video streams over WiMAX is an important challenge, both from the perspective of the Internet Service Provider (ISP), which wants to maximize revenue while keeping customers satisfied, and the customers, which want to have a good service level with the minimum cost. In this context, this paper has presented an experimental performance evaluation of the WirelessMANOFDMA air interface when delivering multiple video streams to different clients. By using a testbed setup and empirical evaluations instead of simulations, no assumptions on the air interface behavior and parameters had to be made, and thus, the results correspond closer to the performance of an actual WiMAX network deployment. The performed study has resorted to Evalvid, a video evaluation framework that allows for the assessment of network and user level performance measures when there is a single client doing video streaming. Since the used experimental setting aims at reproducing real life scenarios as accurately as possible, multiple clients were employed and, thus, a new evaluation methodology was developed to accurately measure the performance of multiple delivered video streams from multiple clients.

By using the developed methodology, experimentation on the testbed was performed with videos which use different compression levels namely, CBR with $256 \mathrm{kbps}$ and $512 \mathrm{kbps}$ and VBR with peak data rate of $540 \mathrm{kbps}$. Network and user level performance of the multiple video streams from the multiple clients was evaluated through the network level parameters, delay and loss, and through the user level assessment metric; MOS. The combined evaluation through the delay, loss and MOS metrics has shown that by using the CBR-256kbps video, it is possible to guarantee a good quality for twice more concurrent streams than with both CBR-512kbps and VBR versions. Moreover, these results highlight the capability of WiMAX using the WirelessMAN-OFDMA air interface to support multi-user video streaming in limit conditions, such as when the bandwidth requirements from the clients exceed the empirically measured maximum capacity of the BS.. Overall, from the view of an ISP, a more conservative compression is able to satisfy a higher number of clients with a reasonable perceived quality, when the same capacity is available. From the user perspective, the quality level obtained with the different compression levels is not significantly affected by using a lower compression. Therefore, the experimental evaluation performed has characterized a win-win 
situation of both the service provider and the service user, for different levels of load in the WiMAX link.

\section{ACKNOWLEDGMENTS}

This work was partially supported by a Short-Term Scientific Mission from the COST Action IC0703 "Data Traffic Monitoring and Analysis (TMA)" and by the European Project FP7-MICIE.

\section{REFERENCES}

[1] IEEE 802.16 Working Group, editor. "IEEE Standard for Local and Metropolitan Area Networks. Part 16: Air Interface for Fixed Broadband Wireless Access Systems. IEEE Std”. 802.16-2004, October 2004

[2] IEEE 802.16 Working Group, editor. "IEEE Standard for Local and Metropolitan Area Networks. Part 16: Air Interface for Fixed Broadband Wireless Access Systems. Amendment 2: Physical and Medium Access Control Layer for Combined Fixed and Mobile Operation in Licensed Bands.” IEEE Std.802.16e-2005, December 2005.

[3] O.I. Hillestad, A. Perkis, V. Genc, S. Murphy, and J. Murphy, "Delivery of on-demand video services in rural areas via IEEE 802.16 broadband wireless access networks" Proceedings of the 2nd ACM international workshop on Wireless multimedia networking and performance modeling, Terromolinos, Spain: ACM, 2006, pp. 43-52.

[4] S.Y. Wang and Y.B. Lin, "NCTUns Network Simulation and Emulation for Wireless Resource Management", Wiley Wireless Communications and Mobile Computing, Vol.5, Issue 8, December 2005, pp. 899-916.

[5] B. Sousa, K. Pentikousis, and M. Curado, "Evaluation of multimedia services in mobile WiMAX," Proceedings of the 7th International Conference on Mobile and Ubiquitous Multimedia, Umeå;, Sweden: ACM, 2008, pp. 64-70.

[6] The Network Simulator - ns-2, Available: $\mathrm{http} / / / \mathrm{www} . i s i . e d u / \mathrm{nsnam} / \mathrm{ns} /$

[7] IEEE, "IEEE Standard for Local and metropolitan area networks- Part 21: Media Independent Handover" IEEE Std 802.21-2008, vol., no., pp.c1-301, Jan. 212009
[8] A. Durantini, M. Petracca, and F. Ananasso, "Experimental Evaluation of IEEE 802.16 WiMAX Performances at 2.5 GHz Band," Wireless Communications and Mobile Computing Conference, 2008. IWCMC '08. International, 2008, pp. 338-343.

[9] K. Pentikousis, J. Pinola, E. Piri, and F. Fitzek, “An experimental investigation of VoIP and video streaming over fixed WiMAX," Modeling and Optimization in Mobile, Ad Hoc, and Wireless Networks and Workshops, 2008. WiOPT 2008. 6th International Symposium on, 2008, pp. 8-15.

[10] K. Pentikousis, J. Pinola, E. Piri, and F. Fitzek, “A measurement study of Speex VoIP and H.264/AVC video over IEEE 802.16d and IEEE 802.11g," Computers and Communications, 2008. ISCC 2008. IEEE Symposium on, 2008, pp. 19-24.

[11] ITU-T Recommendation H.264, "Advanced video coding for generic audiovisual services”, November, 2007.

[12] Kendal Correl. PTP daemon (PTPd). Avaible: http://ptpd.sourceforge.net.

[13] International Electrotechnical Commission, Precision clock synchronization protocol for networked measurement and control systems. Reference numbers: IEC 61588:2004(E), IEEE 1588-2002(E), September 2004

[14] K. Correll, N. Barendt, and M. Branicky. Design Considerations for Software Only Implementations of the IEEE 1588 In Proc. Conference on IEEE 1588, Winterhur, Switzerland, October 2005.

[15] J. Klaue, B. Rathke, and A. Wolisz. "EvalVid - A Framework for Video Transmission and Quality Evaluation", 13th International Conference on Modelling Techniques and Tools for Computer Performance Evaluation, pp. 255-272, Urbana, Illinois, USA, September 2003.

[16] ITU-T Recommendation P.800, "Methods for subjective determination of transmission quality", August, 1996

[17] R. Koenen (Ed.), ISO/IEC, "Overview of the MPEG-4 Standard”, ISO/IEC JTC1/SC29/WG11 N4668, March 2002 